\title{
A finite element analysis on combined convection and conduction in a channel with a thick walled cavity
}

\begin{abstract}
Purpose: The purpose of this paper is to examine the effects of thick wall parameters of a cavity on combined convection in a channel. In other words, conjugate heat transfer is solved.

Design/methodology/approach: Galerkin weighted residual finite element method is used to solve the governing equations of mixed convection.

Findings: The streamlines, isotherms, local and average Nusselt numbers are obtained and presented for different parameters. It is found heat transfer is an increasing function of dimensionless thermal conductivity ratio.

Originality/value: The literature does not have mixed convection and conjugate heat transfer problem in a channel with thick walled cavity.
\end{abstract}

Keyword: Combined convection; Conjugate heat transfer; Nonlinear; Open channel 